\title{
Modeling Study on the Secondary Arc with Stochastic Initial Positions Caused by the Primary Arc
}

\author{
Haoxi Cong, Qingmin Li, Jinyuan Xing and W. H. Siew
}

\begin{abstract}
In the conversion process from primary arc to secondary arc, there exists stochasticness phenomenon of the initial positions of secondary arc. However, the present simulation results of the arcing time with the arc chain model are constant, which is not consistent with the test results. In reaction to the above phenomenon, the stochastic simulation model was first established to calculate the relationship between the conductivity of the air and the temperature. Furthermore, the conductivity along the radius direction of the primary arc was acquired, and then the stochastic initial length of the secondary arc with different primary current was also obtained. Results showed that with the increase of primary current, the average value and dispersion of the initial secondary arc length also increased. Finally, the stochastic model of secondary arc with different initial positions was applied into the arc chain model to calculate the arcing time with dispersion, and the simulation results were compared with the experimental results. Results showed that the simulation results of the arcing time are consistent with the test results, and the relative errors are within $10 \%$, which shows that the stochastic model is effective and reliable.
\end{abstract}

Index Terms-Primary arc; Secondary arc; Initial position; Stochastic model; Arcing time

\section{INTRODUCTION}

$\mathrm{T}$ HE previous field operation experience of power transmission lines have shown that over $90 \%$ of the ground fault is the single-phase ground fault [1]. Thus the timely extinction of secondary arc caused by the single-phase ground fault is important to the success of single-phase reclosing, and it is crucial to the transmission capacity and reliability of the power system [2-4]. To ensure the safe operation of the UHV transmission lines and enhance the stability of the power system, the self-extinction of secondary arc is of the key issues need to be solved.

A large number of tests have been done on the self-extinction of secondary arc both at home and abroad [5-10]. The studies have shown that the arcing time of secondary arc is non-deterministic, with great stochasticness. The experimental test can provide the most direct and effective means to study on the characteristics and physical nature of the secondary arc, and accumulate a large number of reliable basic data. However, it is slightly less flexibility

Manuscript received November 29, 2014; revised March 20, 2015; accepted April 10, 2015. This work was supported by the National Natural Science Foundation of China (51277061 and 51420105011).

Haoxi Cong is with the School of Electrical Engineering, Shandong University, Jinan 250061, China. (E-mail: conghaoxi@163.com)

Qingmin Li and Jinyuan Xing are with the State key Lab of Alternate Electrical Power System with Renewable Energy Sources, North China Electric Power University, Beijing 102206, China. (E-mail: lqmeee@ necpu.edu.cn)

W. H. Siew is with the University of Strathclyde, Glasgow G1 1XW, UK. (E-mail: wh.siew@strath.ac.uk) and difficult to analyze the intrinsic physical mechanism of the secondary arc. With the development of digital computing technologies, the modeling and simulation method has become an important way to study on the self-extinction characteristics of secondary arc. Some experts have been using the simulation method to calculate the arcing time [11-13]. In reference [11], an arc chain model was established and the arcing time was calculated, which showed good consistency with the test results. An electric arc model was further developed in reference [12], which can be used to calculate the arcing time. However, the arcing time obtained from the simulation model would be constant, which is not always consistent with the test data with sensible dispersions. In response to the critical issue, it is necessary to further deepen the simulation arc model studies, which can simulate the arcing time with dispersion.

In this paper, the physical mechanism of the conversion process from primary arc to secondary arc was analyzed, and then the stochastic model of secondary arc with different initial positions was established. The initial position refers to the whole secondary arc tunnel after reformation, including the arc roots and the arc column. After that the relationship between the conductivity of the air and the temperature was acquired, and the conductivity along the radius direction of the primary arc was obtained. Then the stochastic initial length of the secondary arc with different primary current was obtained. Finally, the stochastic model of secondary arc with the initial positions was added into the arc chain model to simulate the dispersive arcing time, and the simulation results were compared with the experimental results.

\section{Modeling AND SimUlation}

\section{A. Generation mechanism of secondary arc}

When there is single-phase ground fault occurred on the power transmission lines, due to the operation delay of the relay protection device and the circuit breaker, the circuit breaker on both sides could not be disconnected immediately. Thus there would be short circuit arc between the failure phase and the ground point, which is called primary arc. Then after dozens of milliseconds, with the action of the relay protection device and the circuit breaker, the failure phase would be cut off. However, due to the capacitance and mutual inductance coupling between the fault phase and non-fault phase, there would still be current flowing through the fault point, which is called secondary arc.

The diameter of the arc is closely related to the current. On the UHV power transmission lines, the primary current can be up to tens of kilo-amperes, which is much larger than the secondary current, and the diameter of primary arc is also much larger than the diameter of secondary arc. There should 
normally be a natural transition from the primary arc to the secondary arc in term of the arc shape, since they are burning in the same arc channel. However, some unique difference still exists between the initial shapes of the primary and secondary arcs especially after the controlled line interruption. Due to higher current and temperature of the primary arc, there would be even larger number of highly ionized particles to form air plasma in the primary arc channel. Just after extinction of the primary arc by deliberated line interruption, the secondary arc would be initialized and develop along the primary arc channel, whose developing direction is normally uncertain and can be calculated based on conductivity distribution. In addition, as the motion behaviour of the primary arc renders randomness, the final shape of the primary arc before extinction is stochastic, hence, the initial shape of the secondary arc turns to be stochastic as well. In other words, the initial positions of secondary arc are stochastic, as shown is Figure 1, in which the dotted lines indicate the possible positions of secondary arc.

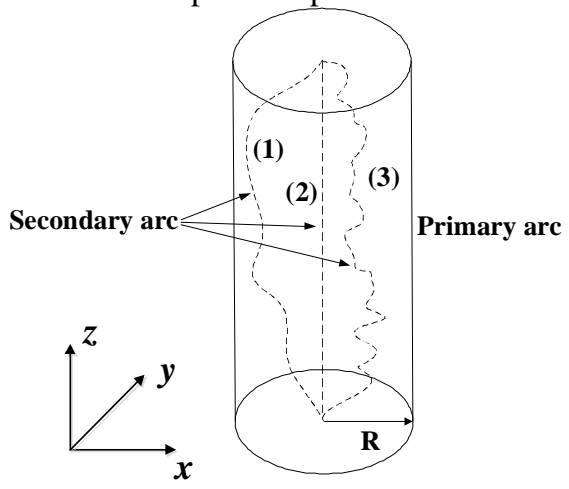

Fig. 1. The stochastic initial positions of secondary arc.

The arc jet from the arc horns of the insulator assemblies may execute impacts on the primary arc motions. However, as the arc jet phenomenon mostly occurs in the arc root area and the outstretched length of the arc column is much longer than that of the arc root for a long gap arc discharge, the randomness of the two arc roots can be neglected during the simulation process. Also, previous studies by other researchers have also shown that, especially in terms of spatial shape and motions of an open air discharge, the dynamics of "long length" and "high current" arcs can be equivalently simulated to some extent by "short length" and "low current" arcs. Hence, in simulating the extinction time of the secondary arcs, low primary arc current is adopted as also to conveniently compare with the experimental results. Moreover, the long gap arcs must meet the mandatory conditions that the arc root are attached to the electrode, thus it can be supposed that the secondary arcs develop from the central location of cathode arc root to the anode arc root along the path of previous primary arc.

\section{B. Conductivity calculation of primary arc}

The developing direction of the arc in air is stochastic, and the stochasticness is related to the air conductivity. The larger the conductivity, the more likely formation of a new arc breakdown. To obtain the conductivity distribution of the arc, some hypotheses are simplified as follows:

(1) The arc column is supposed to be cylindrically symmetric, constant cross section and infinite length;
(2) There is no macroscopic medium flowing between the inside and outside of the arc column;

(3) Within a simulation time step, the conductivity and current density at a certain point inside the arc column is constant.

The diameter of the arc is closely related to the arc current. For the arc burning freely in the air, the diameter $d$ is proportional to the square root of the current $I$ according to the experimental data

$$
d=0.26 \times 10^{-2} \sqrt{I}
$$

where the unit of $d$ is $\mathrm{m}$, and the unit of $I$ is A. $I$ is the root mean square (RMS) value.

According to the above hypotheses, it can be obtained by the Ohm's law $j=\sigma E$ with integrating in the entire channel cross section that

$$
I=2 \pi E \int_{0}^{R} \sigma(T) r d r=\pi R^{2} E \int_{0}^{1} \sigma(x) d x=\pi R^{2} E \int_{T_{W}}^{T_{A}(I)} \sigma(T)\left(-\frac{\partial x}{\partial T}\right) d T
$$

where $I$ is the primary current and the unit is A, $E$ is the electric field strength of the arc column and the unit is $\mathrm{V} / \mathrm{m}, \sigma(T)$ is the conductivity which is the function of temperature and the unit is $\Omega^{-1} \cdot m^{-1}, R=d / 2$ is the radius of the primary arc, $r$ is the radial distance from the center line of the primary arc and the unit is $\mathrm{m}$, $x=(r / R)^{2}, T_{A}$ is the temperature at the center line of the primary $\operatorname{arc}, T_{W}$ is the temperature of the outside surface of the primary arc and the unit is $\mathrm{K}$.

The relationship between the conductivity and the temperature can be obtained by the empirical equations through a lot of experimental statistics [14-16] that

$$
\sigma=a T^{-b} e^{-c / T}
$$

where the constant $a, b$ and $c$ can be obtained through the least squares fitting method with sets of the $E$ and $I$ meet equation (2). The volt-ampere characteristics $E(I)$ and the radial temperature distribution $T(r)$ are from the field data. The unit of variable $\sigma$ is $\Omega^{-1} \cdot m^{-1}$ and the unit of variable $T$ is $\mathrm{K}$.

In the moment of the conversion process from the primary to secondary arc, due to the thermal inertia of gas, the arc temperature does not decrease immediately and is still maintaining a very high value. Thus it can be supposed that the temperature of the primary and the secondary arc is consistent in the conversion moment.

In order to acquire the initial positions of the secondary arc with the above model, the relationship between the conductivity and the temperature of the primary arc need to be got first, namely the parameters $a, b, c$ in equation (3). Four methods were provided in reference [14] and the first method was adopted in this paper. In method 1, the parameters $x$ and $T$ are with approximately linear relationship and $x(T, I)$ can be expressed as

$$
x=\alpha(I) T+\beta(I)
$$

where $\alpha(I), \beta(I)$ are constants related with current, and the unit of variable $T$ is $\mathrm{K}$

With the relationship between the potential gradient $E$ and the arc current $I$ in reference [18] and the relationship between the arc temperature $T$ and the arc current $I$ in references [19-20], also with the simultaneous equations (2), (3), (4), the parameters a, b, c can be obtained. The relationship between the conductivity and the temperature can be expressed as

$$
\sigma=4.77 \times 10^{16} \times T^{-3} \times e^{-8.23 \times 10^{4} / T}
$$


where the unit of variable $\sigma$ is $m \Omega^{-1} \cdot \mathrm{cm}^{-1}$ and the unit of variable $T$ is $\mathrm{K}$.

To verify the accuracy of the calculation, the present results are compared with results in the literature [21], as shown in Figure 2. As can be seen from the figure, the overall trend of the two calculation results is consistent and the deviation is small, which indicate the calculation method is accurate and reliable.

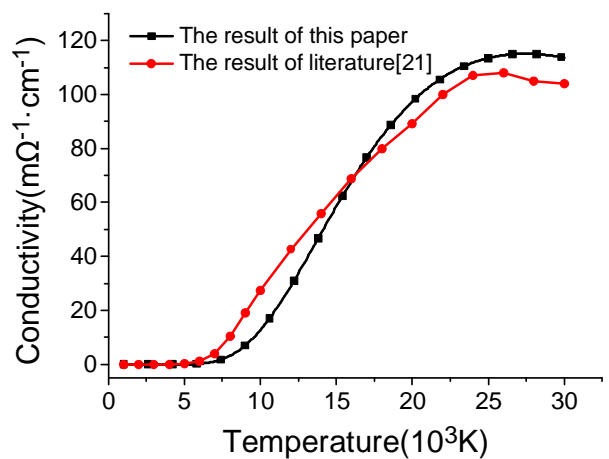

Fig. 2. The relationship between arc conductivity and temperature.

As can be seen from Figure 2, with the increase of temperature, the conductivity is being flat first, afterwards increasing fast and at last being flat again. It is because that in the low temperature stage, the ionization degree of air and the electron density is low, thus the conductivity does not increase obviously. Then with the increase of temperature, the ionization degree of air and the electron density increase correspondingly, thus the conductivity increases too. At last in the high temperature stage, the ionization degree of air and the electron density are very high, the conductivity does not increase even more.

With the relationship between the arc temperature $T$ and the arc current $I$ in references [19], the center temperature of the arc can be obtained with primary current of $1 \mathrm{kA}$ and $25 \mathrm{kA}$. If the surface of the arc was set to $2000 \mathrm{~K}$, and with the simultaneous equations of (1), (4), (5), the conductivity distribution along the radial direction of primary arc can be acquired, as shown in Figure 3.

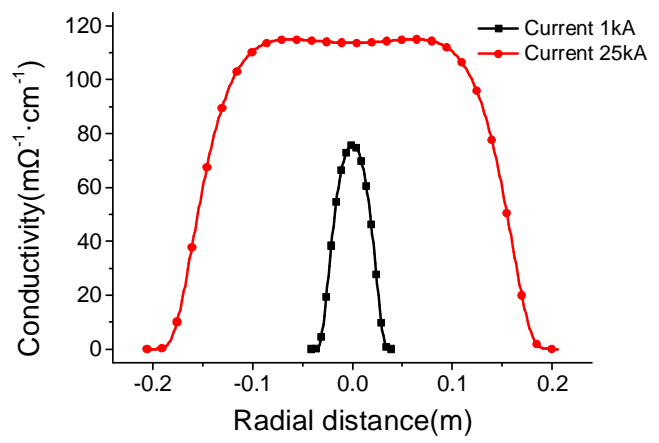

Fig. 3. The conductivity distribution of primary arc along the radial direction.

\section{Developing direction of secondary arc}

As the electron flow of the arc is mostly emitted by the cathode itself, the particle density is the largest and the motion speed of the electrons is the highest, therefore the entire course of the cathode is the most significant to the whole conversion process of the arc. Previous studies have found that the temperature of the cathode arc root is the highest, then comes the temperature of the arc column, and finally the anode arc root. $[17,20]$ Therefore in the model, the secondary arc is supposed to develop from the cathode arc root to the anode arc root. The initial position is supposed to be at the center position of the primary cathode arc root and the developing direction was the whole half sphere, as shown in Figure $4 . \beta$ is the included angle between the developing direction of the secondary arc and the positive direction of $z$-axis, $\alpha$ is the angle between the projection of the secondary arc on $x y$ plane and the positive direction of $x$-axis. The range of $\alpha$ is $0 \sim 360^{\circ}$ and the range of $\beta$ is $0 \sim 90^{\circ}$.

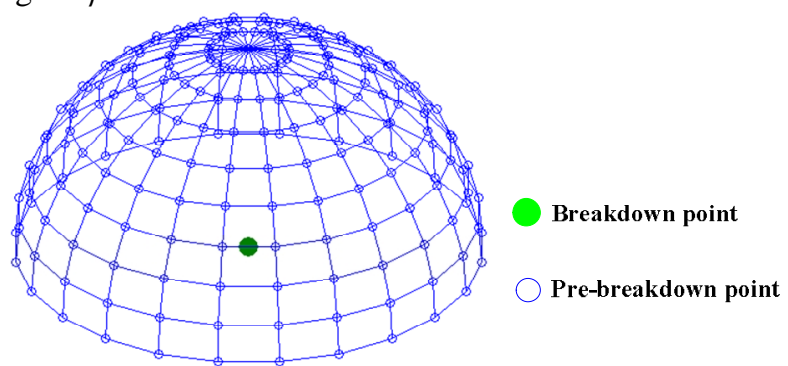

(a) The possible breakdown point of next step

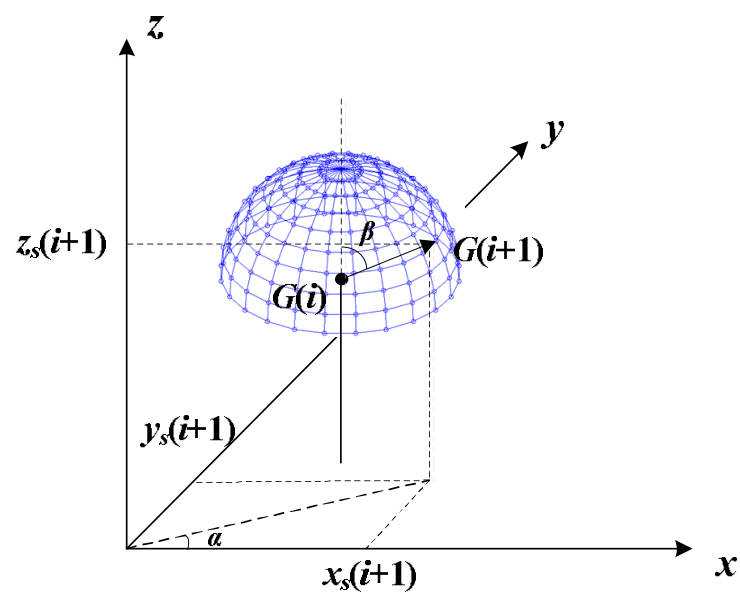

(b) The coordinates of the pre-breakdown point

Fig. 4. The stochasticness in the development of the secondary arc.

The coordinates of the breakdown point and pre-breakdown point can be expressed as

$$
\left\{\begin{array}{l}
x_{s}(i+1)=x_{s}(i)+r_{s} \cdot \sin \beta \cdot \cos \alpha \\
y_{s}(i+1)=y_{s}(i)+r_{s} \cdot \sin \beta \cdot \sin \alpha \\
z_{s}(i+1)=z_{s}(i)+r_{s} \cdot \cos \beta
\end{array}\right.
$$

in which $x_{s}(i), y_{s}(i), z_{s}(i)$ is the coordinates of the breakdown point, $x_{s}(i+1), y_{s}(i+1), z_{s}(i+1)$ is the coordinates of the pre-breakdown point, $r_{s}$ is the calculation step length. Here the unit of $x_{s}(i), y_{s}(i), z_{s}(i)$ and $r_{s}$ is $\mathrm{m}$.

The conductivity of each position can be obtained through the equations (1)-(6). As the temperature distribution is not uniform along the radial direction, the possibility of the arc developing directions is not same. Therefore, in this model, the conductivity of each developing direction is accumulated as the possible coefficients of each developing direction, and the 
probability of each developing directions can be expressed as

$$
P(i, j)=\sigma(i, j) / \sum \sum \sigma(i, j)
$$

where $\sigma(i, j)$ is the conductivity of each developing direction, $P(i, j)$ is the probability of each developing direction.

When the distance between the developing position of arc and the anode is less than a critical length, there would be short circuit between them, which can be expressed as

$$
\sqrt{\left(x_{s}-x\right)^{2}+\left(y_{s}-y\right)^{2}+\left(z_{s}-z\right)^{2}} \leq l_{s}
$$

where $x_{s}, y_{s}, z_{s}$ is the position coordinates of arc, $x, y, z$ is the coordinates of anode, $l_{s}$ is the critial length. In simulation, it can be set that $l_{s}=R$.

\section{The simulation flowchart}

With the establishment of the above model, the simulation flowchart can be represented as follows.

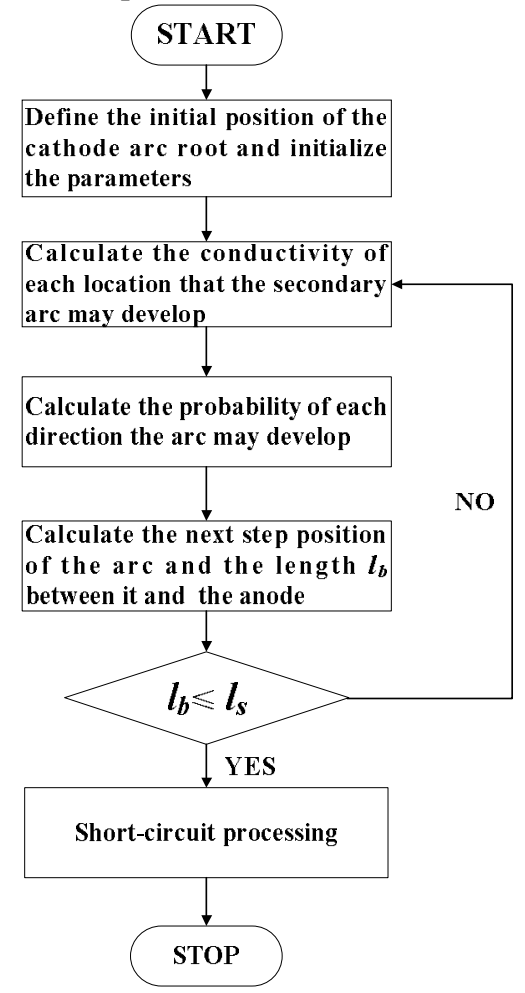

Fig. 5. The simulation process of the secondary arc initial positions.

\section{INITIAL LENGTH Stochasticness OF SECONDARY ARC}

The test results of long gap arcs have shown that, the arc length changes greatly during the arcing process, which may produce great influence on the self-extinction characteristics [22-23]. The arc length is an important parameter during the arc motion process. As the stochasticness of secondary arc can be reflected by the initial length in a certain extent, thus the initial length was used in this paper to study on the stochasticness of secondary arc.

The primary arc was supposed to be standard cylindrical, and its diameter was determined by the primary current. The initial position of secondary arc can be obtained by the established model with different primary currents. For ease of calculation, the gap length of the insulator assemblies was set to be $1 \mathrm{~m}$.

The initial position of secondary arc when the primary current is $1 \mathrm{kA}$ is shown in figure 6(a) while the initial position of secondary arc when the primary current is $25 \mathrm{kA}$ is shown in figure $6(\mathrm{~b})$. And they are compared in figure 6(c).

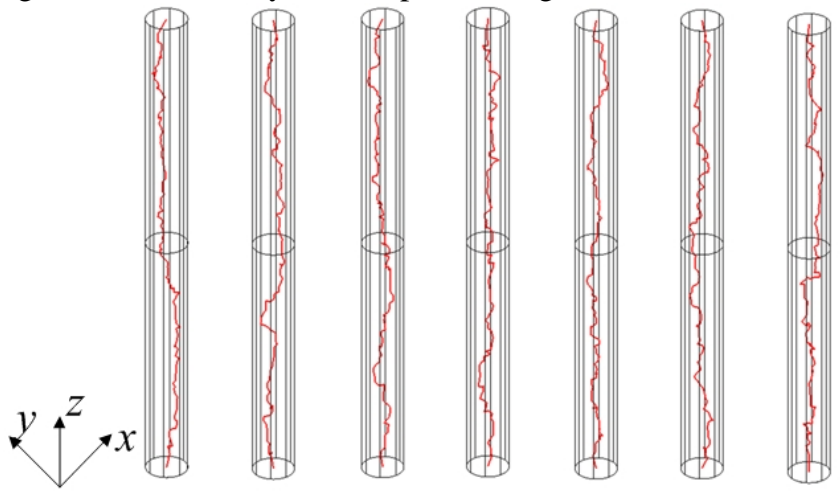

(a) Secondary arc position with primary current $1 \mathrm{kA}$

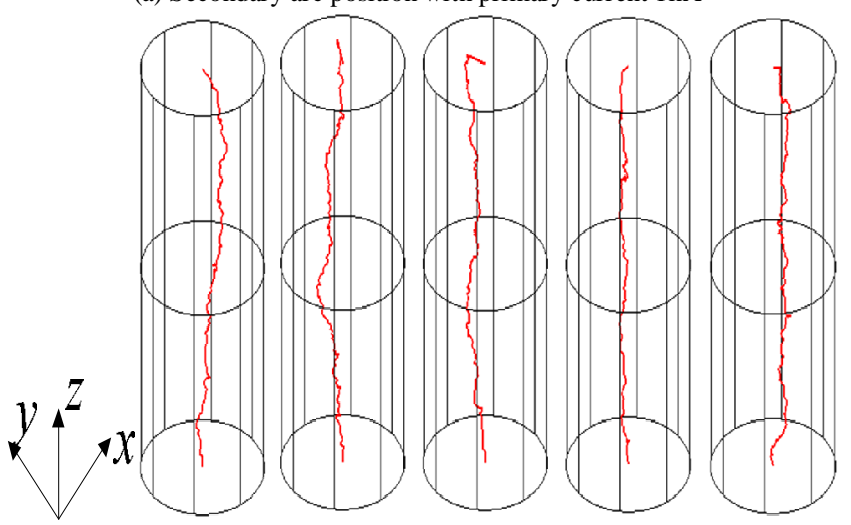

(b) Secondary arc position with primary current $25 \mathrm{kA}$

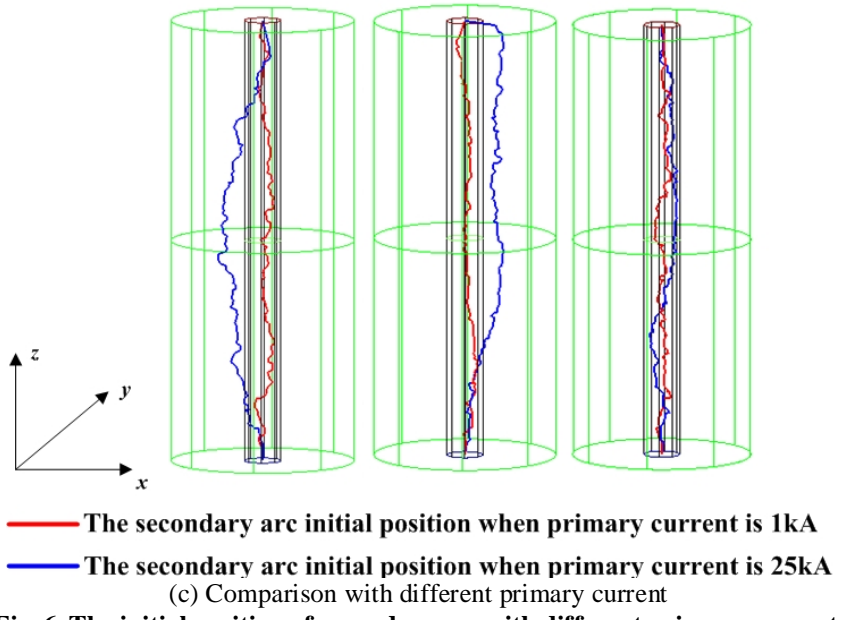

Fig. 6. The initial position of secondary arc with different primary current.

Due to the influence of primary arc, the initial position of secondary arc is stochastic. As can be seen from Figure 6(a) and Figure 6(b), the initial position of secondary is stochastic, and they are developing within the primary arc path. It can be seen from Figure 6(c) that with the increase of primary current, the stochasticness of initial position of primary arc also increases and the arc motion is more complex.

In order to analyze the influence of different primary current on the initial position of secondary arc, the initial length of secondary arc are calculated with different primary current. Each set has 25 valid data, as shown in Table I. Here, the gap 
length of the insulator assemblies is set to be $1 \mathrm{~m}$, and the secondary arc value is set to be $30 \mathrm{~A}$.

TABLE I

THE INITIAL SECONDARY ARC LENGTH WITH DIFFERENT PRIMARY CURRENT

\begin{tabular}{ccccc}
\hline \hline $\begin{array}{c}\text { Primary } \\
\text { current/kA }\end{array}$ & $\begin{array}{c}\text { Average arc } \\
\text { length/m }\end{array}$ & Maximum/m & Minimum/m & $\begin{array}{c}\text { Standard } \\
\text { Deviation }\end{array}$ \\
\hline 1 & 1.343 & 1.43 & 1.24 & 0.0531 \\
5 & 1.389 & 1.47 & 1.26 & 0.0540 \\
10 & 1.44 & 1.51 & 1.28 & 0.0543 \\
15 & 1.423 & 1.54 & 1.30 & 0.0639 \\
20 & 1.465 & 1.57 & 1.31 & 0.0725 \\
25 & 1.476 & 1.63 & 1.31 & 0.0976 \\
\hline \hline
\end{tabular}

As can be seen from Table I, with the increase of primary current, the initial length and dispersion of secondary arc increase too. This is due to that with the increase of primary current, the diameter of primary arc increases correspondingly. Theoretically, the secondary arc can develop anywhere along the path of primary arc, thus the initial length and dispersion of secondary arc increases with the increasing of primary current. It can be also seen from the table that, with the increase of primary arc current, the maximum value of the initial length of the secondary arc increases correspondingly while there is little change of the minimum length. It was because that the secondary arc developed along the path of primary arc and theoretically the initial length is equal to the primary arc length. As long as the sample is large enough, the rule can be discovered.

\section{ARCing Time Stochasticness of Secondary ARC}

The actual shape of arc is complex and constantly varying over time, thus it cannot be simplified into a single cylinder. Therefore the arc chain model which can reflect the actual shape changes of long gap arc was adopted [13,24], as shown in Figure 7(a). The secondary arc was suffering the combined action of electromagnetic force, thermal buoyancy force, wind load and air resistance during the motion process. With the arc chain model, the force of secondary arc can be transformed into each current element, as shown in Figure 7(b).

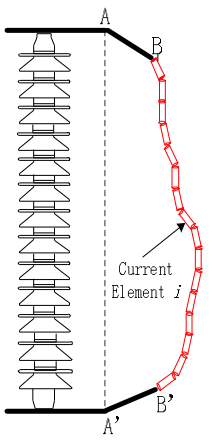

(a)

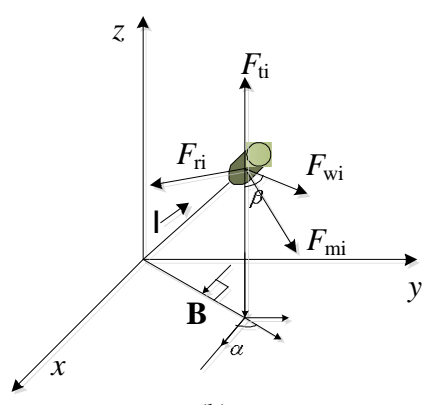

(b)
Fig. 7. Cascade chain model for secondary arcs.

The magnetic force of each current element $i$ can be expressed as

$$
\boldsymbol{F}_{m i}=l_{a i} \boldsymbol{I}_{a i} \times \boldsymbol{B}_{i}
$$

where $l_{a i}$ is the length of the $i$ th current element, $\boldsymbol{I}_{a i}$ is the current vector of the $i$ th current element. $\boldsymbol{I}_{a i}$ is the root mean square
(RMS) value. The unit of $\boldsymbol{F}_{\mathrm{mi}}$ is $\mathrm{N}$, the unit of $l_{a i}$ is $\mathrm{m}$, the unit of $\boldsymbol{I}_{a i}$ is A, and the unit of $\boldsymbol{B}_{i}$ is T.

The current element will be subjected to the vertical thermal buoyancy which can be derived according to buoyancy equation as

$$
F_{t i}=\left(\rho_{0}-\rho\right) \cdot g \pi r_{a i}^{2} l_{a i}
$$

where $\rho_{0}$ is the air density under standard atmospheric pressure and temperature with a typical value of $1.293 \mathrm{~kg} / \mathrm{m}^{3}$. With consideration of the primary arc, $\rho$ denotes the air density in the hot zone around the arc column with a value of $0.0221 \mathrm{~kg} / \mathrm{m}^{3}, r_{a i}$ is the radius of the arc and $r_{a i}=0.0013 \sqrt{I}$, and $\mathrm{g}=9.8 \mathrm{~m} / \mathrm{s}^{2}$ is the gravity acceleration. The unit of $\boldsymbol{F}_{\mathrm{ti}}$ is $\mathrm{N}$ and the unit of $r_{a i}$ is m.

The wind load $\boldsymbol{F}_{w i}$ acting on the $i$ th current element can be expressed as the following expression

$$
\boldsymbol{F}_{w i}=0.72 \cdot r_{a i} l_{a i} \cdot \rho \boldsymbol{v}_{w}^{2}
$$

where $\boldsymbol{v}_{w}$ is the wind speed. The unit of $\boldsymbol{F}_{\mathrm{wi}}$ is $\mathrm{N}$ and the unit of $\boldsymbol{v}_{w}$ is $\mathrm{m} / \mathrm{s}$.

During the motion of the secondary arc, the wind direction and its speed can be supposed to remain the same as the arc motion time is very short. As shown in Fig. 7(b), the x-axis direction is the axial direction of the transmission wires, the $\mathrm{z}$-axis is the direction along the insulator string, $\alpha$ is the angle between the projection of wind in $x y$ plane and positive y-axis, $\beta$ is the angle between the wind and positive $z$-axis, therefore the projection of wind load in the $x, y$, and $z$ axis can be described as

$$
\left\{\begin{array}{l}
F_{w i_{-} x}=F_{w i} \cdot \sin \beta \cdot \cos \alpha \\
F_{w i_{-} y}=F_{w i} \cdot \sin \beta \cdot \sin \alpha \\
F_{w i_{-} z}=F_{w i} \cdot \cos \beta
\end{array}\right.
$$

According to the aerodynamic theory, the air resistance force applied to the $i$ th current element can be expressed as

$$
\boldsymbol{F}_{a i}=C_{r} \cdot r_{a i} l_{a i} \cdot \rho \boldsymbol{v}_{i}^{2}
$$

where $C_{\mathrm{r}}$ is the air resistance coefficient with a value of $1.18 . v_{\mathrm{i}}$ is the motion speed of current element. The unit of $\boldsymbol{F}_{\mathrm{wi}}$ is $\mathrm{N}$ and the unit of $v_{i}$ is $\mathrm{m} / \mathrm{s}$.

The current element is under the combined action of the electromagnetic force, the thermal buoyancy, the wind loads and the air resistance. The mass density of the current element is far less than that of the air, so its quality and acceleration process can be neglected, the movement velocity is determined by these four forces as

$$
\boldsymbol{F}_{m i}+\boldsymbol{F}_{t i}+\boldsymbol{F}_{a i}+\boldsymbol{F}_{w i}=m_{i} \boldsymbol{a}=\mathbf{0}
$$

Thus, the secondary arc speed can be acquired from equation (9) (14)

$$
\left\{\begin{array}{l}
v_{i_{-} x}=\sqrt{\frac{F_{m i_{-} x}}{C_{r} r_{i} L_{i} \rho_{0}}+f_{r} \cdot v_{w}{ }^{2} \cdot \sin \beta \cdot \cos \alpha} \\
v_{i_{-} y}=\sqrt{\frac{F_{m i_{-} y}}{C_{r} r_{i} L_{i} \rho_{0}}+f_{r} \cdot v_{w}{ }^{2} \cdot \sin \beta \cdot \sin \alpha} \\
v_{i_{-} x}=\sqrt{\frac{F_{m i_{-} x}+\left(\rho_{0}-\rho\right) g \pi r_{i}{ }^{2} L_{i}}{C_{r} r_{i} L_{i} \rho_{0}}+f_{r} \cdot v_{w}{ }^{2} \cdot \cos \beta}
\end{array}\right.
$$

where $v_{a i_{-} x}, v_{a i_{-} y}, v_{a i_{z} z}$ is the speed of the $i$ th current element in $x, y, z$ direction, $F_{m i_{-}}, F_{m i-y}, F_{m i_{-} z}$ respectively denote the $x$, $y, z$ directional components of the magnetic force, and 
$f_{r}=0.72 / C_{\mathrm{r}}$.

With the arc chain model, the motion trajectory of the secondary arc can be calculated and the arc length is also obtained. The arc length $l$ is used as the self-extinction criterion of the secondary arc, which is derived from statistics of a lot of experimental data. The expression of the critical self-extinction length $l_{\max }$ is given by

$$
l_{\max }=0.7 I_{a m}^{0.25} U_{a m} \times 10^{-4}
$$

where $I_{a m}$ and $U_{a m}$ are the peak values of the secondary current and the supply voltage. The unit of $l_{\max }$ is $\mathrm{m}$, the unit of $I_{a m}$ is A, and the unit of $U_{a m}$ is $\mathrm{V}$.

Based on the stochastic initial length of secondary arc, the stochastic model was applied into the arc chain model to simulate the arcing time of secondary arc. The simulation process can be categorized into three steps: a) The arc chain model is used to calculate the motion process of primary arc. b) The established stochasitc model is used to calculate the initial positions of secondary arc. c) The arc chain model is used to calculate the motion process of secondary arc. In this paper, the physical simulation test of secondary arc on transmission lines was taken as the research object. The primary arc is set to $1 \mathrm{kA}$, the secondary arc current is $30 \mathrm{~A}$, and the length of the insulation string is $0.68 \mathrm{~m}$. Figure 8 (a) shows five typical results of the stochastic initial positions of the secondary arcs based on simulation analysis. Different initial positions of the secondary arcs will result in different motion trajectories of the secondary arcs. Figure 8(b) gives the secondary arc trajectories before the extinction instant regarding the five typical cases.

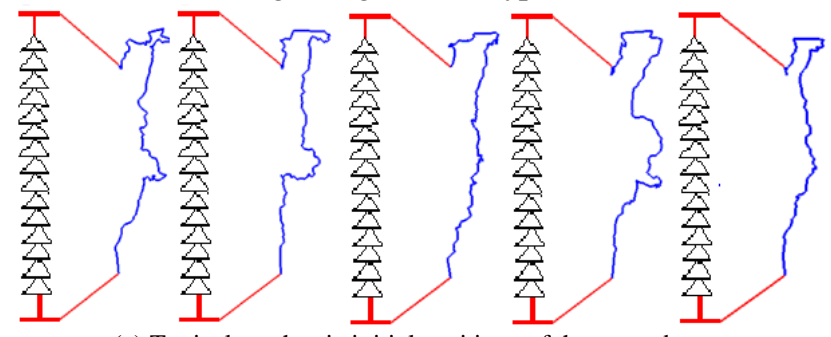

(a) Typical stochastic initial positions of the secondary arc

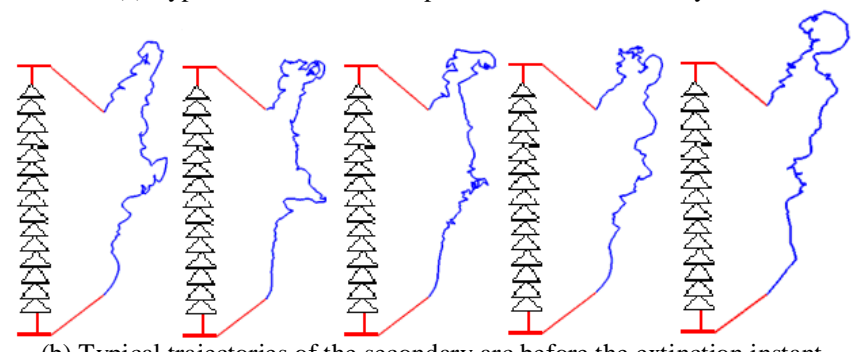

(b) Typical trajectories of the secondary arc before the extinction instant Fig. 8. Stochastic initial positions and trajectories of the secondary arc before the extinction instant.

As can be seen from Figure 8(a), with consideration of the stochastic model in the arc chain simulation, the initial positions of the secondary arc are different, which result in different motion trajectories of the secondary arcs. As can be seen from Figure 8(b), the secondary arc trajectories vary greatly before its extinction, reflecting the impacts of different initial positions on the motion trajectories.

An experimental platform is established to study the arcing time of the secondary arc discharge, as is shown in Figure 9(a) and 9(b). The platform is mainly composed of a test circuitry together with a corresponding measurement system, including a $11.6 \mathrm{kV}$ AC power supply, group capacitors, copper electrodes, HV insulators and metal fuses to ignite the arc discharge. Here, $L$ is used to denote the equivalent inductance of the transmission line, and $C$ is used to simulate the equivalent coupling capacitance between the faulty phase and healthy phases. Here $L=0.03688 \mathrm{H}$, and different secondary arc currents are achieved by changing the values of the group capacitors $C$. The gap length of the insulator assemblies is $0.68 \mathrm{~m}$.

The operation procedure of the experiment is shown as follows. Firstly, the circuit breaker $\mathrm{CB}_{1}$ is closed to produce an inductive primary arc current, and the triggered arc wire will form an arc channel due to gasification effect of the primary current at about $1 \mathrm{kA}$. Secondly, the circuit breaker $\mathrm{CB}_{2}$ is closed after $0.1 \mathrm{~s}$ and $\mathrm{CB}_{1}$ is quickly opened to ignite and simulate the secondary arc current. The arc trajectories are recorded by a high speed video camera, while the voltage and current of the secondary arcs are measured through voltage dividers, CTs and digital oscilloscopes. To avoid dispersion, fifteen data sets are obtained for each case of the test scheme. Figure 9(c) is the high speed arc images recorded by the high speed video camera.

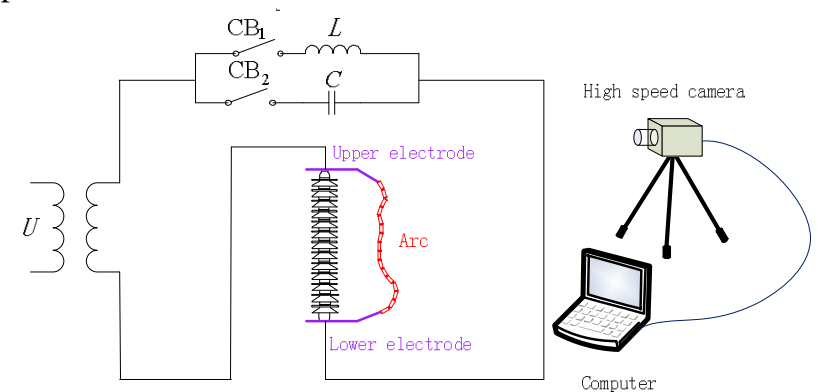

(a) The single-phase physical simulation circuitry
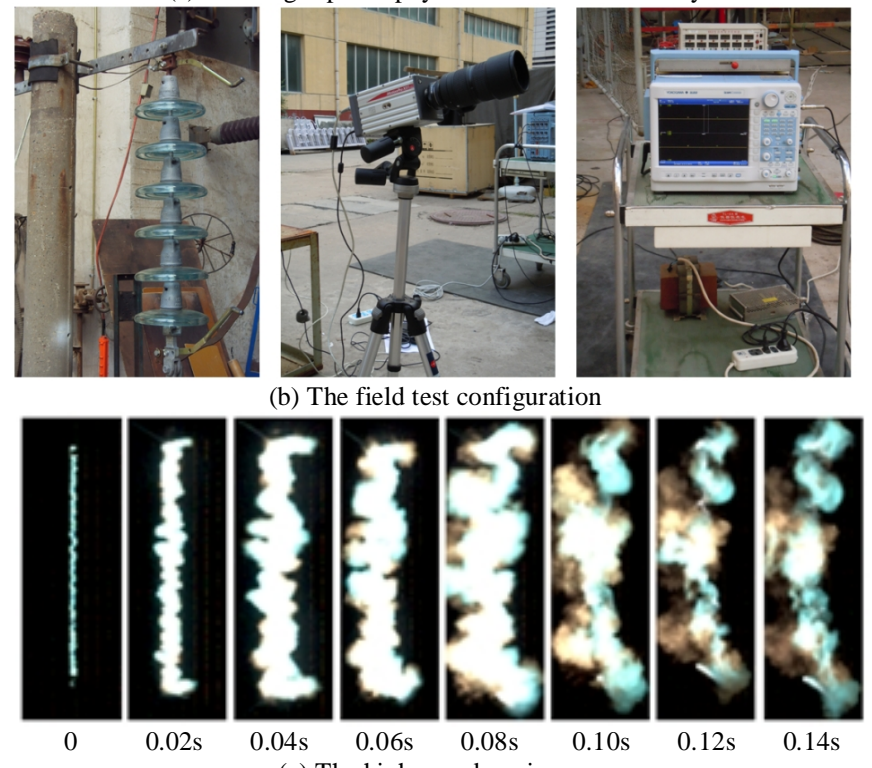

(c) The high speed arc image

Fig. 9. The established experimental platform and high speed arc image.

The experiment shows that in the arc transition moment, the initial positions of the secondary arc are all in the whole channel of the primary arc. As can be seen from Figure 9(c), at the time instant of $0.1 \mathrm{~s}$, the primary arc extinguished and the 
secondary arc started. The secondary arc is completely burning in the inner part of the primary arc plasma. The different initial positions of secondary arc would result in dispersion of the arcing time. The arcing time is entitled to the time the secondary arc lasts before extinction. The arcing time was calculated with different secondary current and each set has 30 valid data. The results are compared with the test results, as shown in Figure 10. The arcing time with different secondary current level is given in Table II, and the arc elongation with different secondary current level is shown in Table III.

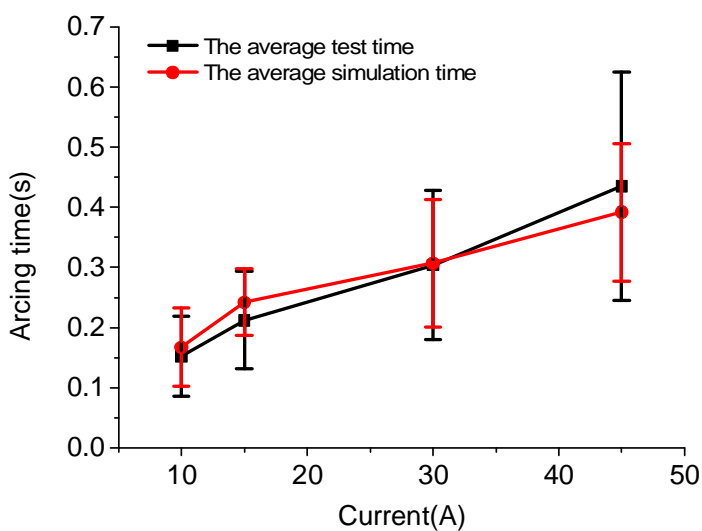

Fig. 10. The dispersion of arcing time between test and simulation data.

TABLE II

ARCING TIME OF SECONDARY ARC UNDER DIFFERENT SECONDARY CURRENT

\begin{tabular}{ccccccc}
\hline \hline $\begin{array}{c}\text { Secondary } \\
\text { current/A }\end{array}$ & Test time/s & $\begin{array}{c}\text { Average } \\
\text { value/s }\end{array}$ & $\begin{array}{c}\text { Standard } \\
\text { deviation }\end{array}$ & $\begin{array}{c}\text { Simulation } \\
\text { time/s }\end{array}$ & $\begin{array}{c}\text { Average } \\
\text { value/s }\end{array}$ & $\begin{array}{c}\text { Standard } \\
\text { deviation }\end{array}$ \\
\hline 10 & $\begin{array}{c}0.056 \sim 0.3 \\
54\end{array}$ & 0.152 & 0.0661 & $\begin{array}{c}0.054 \sim 0.2 \\
82\end{array}$ & 0.167 & 0.0653 \\
& $\begin{array}{c}0.094 \sim 0.3 \\
68\end{array}$ & 0.212 & 0.081 & $\begin{array}{c}0.084 \sim 0.3 \\
06\end{array}$ & 0.242 & 0.0556 \\
& $\begin{array}{c}6.096 \sim 0.6 \\
25\end{array}$ & 0.304 & 0.1245 & $\begin{array}{c}0.116 \sim 0.4 \\
86\end{array}$ & 0.307 & 0.1065 \\
& $\begin{array}{c}250 \\
45\end{array}$ & 0.435 & 0.1895 & $\begin{array}{c}0.244 \sim 0.7 \\
02\end{array}$ & 0.392 & 0.1144 \\
\hline \hline
\end{tabular}

TABLE III

ELONGATION OF SECONDARY ARC UNDER DIFFERENT SECONDARY CURRENT

\begin{tabular}{cccccc}
\hline \hline $\begin{array}{c}\text { Secondary } \\
\text { current/A }\end{array}$ & $l_{1} / \mathrm{m}$ & $l_{2} / \mathrm{m}$ & $l_{3} / \mathrm{m}$ & $\triangle l_{1} \%$ & $\triangle l_{2} \%$ \\
\hline 10 & 0.98 & $0.86-2.10$ & 2.23 & $128 \%$ & $6 \% \sim 159 \%$ \\
15 & 0.98 & $0.84-2.22$ & 2.46 & $151 \%$ & $11 \% \sim 193 \%$ \\
30 & 0.98 & $0.90-2.34$ & 2.93 & $199 \%$ & $25 \% \sim 226 \%$ \\
45 & 0.98 & $0.89-2.30$ & 3.24 & $231 \%$ & $41 \% \sim 264 \%$ \\
\hline \hline
\end{tabular}

$l_{1}$ is the initial length of the secondary arc without stochasticness, $l_{2}$ is the initial length of secondary arc with stochasticness, $l_{3}$ is the arc length before the extinction instant. $\triangle l_{1} \%$ is the arc elongation of $l_{1}$, and $\triangle l_{2} \%$ is the arc elongation of $l_{2}$.

It can be seen from Table II that the arcing time of secondary arc increases along with the rise of secondary current. It can be seen from Table III that, there exists a variation range of the arc elongation according to the adopted stochastic model. The overall trend of the simulation results is consistent with the experimental ones, indicating that the proposed model is effective and reliable. It can be also found that the dispersion of calculation time is less than the test time, which is due to that only the stochastic initial position of secondary arc is consider in the stochastic model while in the test, the stochasticness of external environment also has an impact on the arcing time. It can be also found in Table II that both in the simulation and test, with the increase of secondary current, the arcing time increase too.

\section{CONCLUSIONS}

1) The stochastic simulation model of secondary arc with different initial positions was established, which suppose that the initial position of secondary arc is stochastic and the stochasticness is related to the conductivity.

2) With the stochastic simulation model, the relationship between the conductivity and temperature of primary arc was obtained and the conductivity along the radial direction of primary arc was further acquired.

3) The stochastic initial positions of secondary arc with different primary current were simulated. Results showed that with the increase of primary current, the initial length and dispersion of secondary arc also increased.

4) The stochastic simulation model was applied into the arc chain model to simulate the arcing time of secondary arc. The simulation results were compared with the test results. The overall trend between them is consistent and the relative error is within $10 \%$, indicating the stochastic model is effective and reliable.

\section{ACKNOWLEDGEMENT}

Financial support by the National Natural Science Foundation of China (51277061 and 51420105011) is here acknowledged.

\section{REFERENCES}

1] IEEE Power system relaying committee working group, "Protective relaying performance reporting," IEEE Transactions on Power Delivery, vol. 7, no. 4, pp. 1892-1899, 1992.

[2] T. Tsuboi, J. Takami, S. Okabe, K. Aoki, and Y. Yamagata, "Study on a Field Data of Secondary Arc Extinction Time for Large-sized Transmission Lines," IEEE Transactions on Dielectrics and Electrical Insulation, vol. 20, no. 6, pp. 2277-2286, 2013.

[3] Z. Y. Xu, X. Zhang, X. Q. Yan, and A. Wen, "Optimal compensating scheme for limiting secondary arc current of 1000-kV UHV short parallel lines," IET Generation Tranmission \& Distribution, vol. 6, no. 12, pp. 1235-1242, 2012.

[4] Qingmin Li, Qiuqin Sun, Jie Lou, Li Zhang, Haoxi Cong, "Research on a novel suppressing methodology for secondary arc extinction based on an impedance paralleled to line circuit breaker," International Transactions on Electrical Energy Systems, vol. 24, no. 2, pp. 281-296, 2014.

[5] I. M. Dudurych, T. J. Gallagher, E. Rosolowski, "Arc effect on single-phase reclosing time of a UHV power transmission line," IEEE Transactions on Power Delivery, vol. 19, no. 2, pp. 854-860, 2004.

[6] Yutaka Goda, Shoji Matsuda, Tsuginori Inaba, "Forced extinction characteristics of secondary arc on UHV transmission lines," IEEE Transactions on Power Delivery, vol. 8, no. 3, pp. 1322-1330, 1993.

[7] A. A. Montanari, M. C. Tavares, C. M. Portela, "Secondary arc voltage and current harmonic content for field tests results," Proceedings of the 2009 International Conference on Power Systems Transients, Kyoto, Japan, June 2009.

[8] Z. G. Xu, X. Q. Yan, X. Zhang, and A. Wen, "Compensating scheme for limiting secondary arc current of $1000 \mathrm{kV}$ ultra-high voltage long parallel lines," IET Generation Tranmission \& Distribution, vol. 7, no. 1, pp. 1-8, 2013.

[9] Virginie Inguimbert, Daniel Sarrail, and Jean-Charles Mateo-Velez, "Electrostatic Discharge and Secondary Arcing on Solar Array-Flashover Effect on Arc Occurrence," IEEE Transactions on Plasma Science, vol. 36, no. 5, pp. 2404-2412, 2008

[10] He Yanmiao, Song Gao, Cao Rongjiang, "Test Research of Secondary Arc in $1000 \mathrm{kV}$ UHV Double-circuit Transmission Lines," Proceedings of the CSEE, vol. 31, no. 16, pp. 138-143, 2011. 
[11] V. V. Terzija, H. J. Koglin, "On the modeling of long arc in still air and arc resistance calculation," IEEE Transactions on Power Delivery, vol. 19, no. 3, pp. 1012-1017, 2004.

[12] S. Q. Gu, J. L. He, B. Zhang, G. Z. Xu, and S. J. Han, "Movement simulation of long electric arc along the surface of insulator string in free air," IEEE Transactions on Magnetics, vol. 42, no. 4, pp. 1359-1362, 2006.

[13] Qingmin Li, Haoxi Cong, Qiuqin Sun, Jinyuan Xing and Quan Chen, "Characteristics of Secondary AC Arc Column Motion near Power Transmission Line Insulator String," IEEE Transactions on Power Delivery, vol. 29, no. 5, pp. 2324-2331, 2014

[14] Devoto R S, Mukherjee D, "Electrical conductivity from electric arc measurements,"Journal of Plasma Physics, vol. 9, no. 1, pp. 65-76, 1973.

[15] Bauder U, Devoto R S, Mukherjee D, "Measurement of electrical conductivity of argon at high pressure," Physics of Fluids, vol. 16, no. 12, pp. 2143-2148, 2003.

[16] Devoto R S, Bauder U H, Cailleteau J, "Air transport coefficients from electric arc measurements," Physics of Fluids, vol. 21, no. 4, pp. 552-558, 2008.

[17] S. Q. Gu, J. L. He, R. Zeng, B. Zhang, G. Z. Xu, and W. J. Chen, "Motion characteristics of long ac arcs in atmospheric air," Applied Physics Letters, vol. 90, no. 5, pp. 051501, 2007.

[18] Gu Jinguo, Xu Guozheng, Qian Jiali, "Characteristics of Fault Arc," High Voltage Apparatus, vol. 35, no. 6, pp. 41-44, 1999.

[19] Guo Zengyuan, Zhao Wenhua, Arc and Thermal Plasma, pp. 67-68, Science Press, China.

[20] Yan Xianglian, Chen Wweijiang, He Ziming, "Temperature Measurement of Air Arc in Long Gap by Spectrum Diagnosis," Proceedings of the CSEE, vol. 31, no. 19, pp. 146-152, 2011.

[21] J. M. Yos, Technical Memorandum RAD-TM-63-7, AVCO Corporation, Wilmington (MA), 1963.

[22] Haoxi Cong, Qingmin Li, Jinyuan Xing, and Jinsong Li, "Typical motion and extinction characteristics of the secondary arcs with half-wavelength transmission line," Plasma Science \& Technology, vol. 16, no. 9, pp. 843-847, 2014.

[23] G. B. Santos, C. L. Tozzi, and M. C. Tavares, "Visual Evaluation of the Length of Artificially Generated Electrical Discharges By 3D-snakes," IEEE Transactions on Dielectrics and Electrical Insulation, vol. 18, no. 1, pp. 200-210, 2011.

[24] K. Horinouchi, Y. Nakayama, M. Hidaka, T. Yonezawa and H. Sasao, “A method of simulating magnetically driven arcs [in switchgear]," IEEE Transactions on Power Delivery, vol. 12, no. 1, pp. 213-218, 1997.

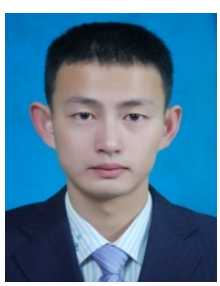

Haoxi Cong received the B.Sc. (Hons.) degree in electrical engineering from Shandong University, Jinan, China, in 2011, where he is currently pursuing the Ph.D. degree in electrical engineering. His research interests are secondary arcs with half-wavelength transmission lines and the interaction mechanism with the electromagnetic transients of power systems.

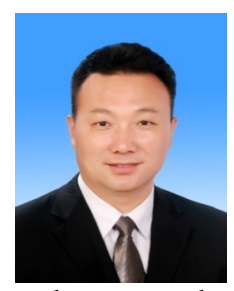

Qingmin Li is now a professor in electrical engineering at North China Electric Power University, China. He graduated from Tsinghua University, China, where he got his degrees of BSc (1991), MSc (1994) and PhD (1999) in electrical engineering. He joined Tsinghua University as a lecturer in 1996, and came to the UK in 2000 as a postdoctoral research fellow working at Liverpool University and later at Strathclyde University. He joined Shandong University in 2003 and worked there as a professor in electrical engineering till 2011. He also worked at Arizona State University, USA, as a visiting professor from February to August 2010. His special fields of interest include high-voltage engineering, applied electromagnetics, condition monitoring and fault diagnostics, high-voltage power electronics, etc. He is a member of IET and IEEE.

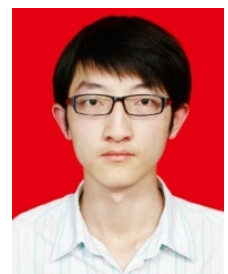

Jinyuan Xing received the B.Sc. degree in electrical engineering from Jilin University, Changchun City, China, in 2012 and is currently pursuing the M.Sc. degree in electrical engineering from North China Electric Power University, Beijing, China, with a specific research interest in secondary arcs with half-wavelength transmission lines.

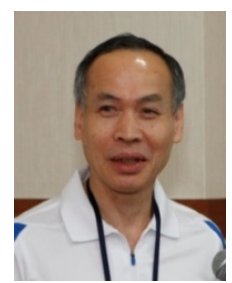

W. H. Siew is a Reader in the Department of Electronic \& Electrical Engineering, University of Strathclyde, Glasgow, Scotland. He is a triple alumnus of the University of Strathclyde with degrees of B.Sc. (Hons) in electronic and electrical engineering; Ph.D. in electronic \& electrical engineering; and Master of Business Administration. His areas of research interest include large systems electromagnetic compatibility, cable diagnostics, lightning protection and wireless sensing systems. He is Convener of the CIGRE WG C4.208 and a member of the Technical Advisory Panel for the IET Professional. 